\title{
Retraction Note to: Image Colorization Using Convolutional Neural Network
}

\author{
Yili Zhao ${ }^{1,2(\bowtie)}$, Dan $\mathrm{Xu}^{1}$, and Yan Zhang $^{2}$ \\ 1 Yunnan University, Kunming 650091, Yunnan, China \\ ylzhao@vip.sina.com, danxu@ynu.edu.cn \\ ${ }^{2}$ Southwest Forestry University, Kunming 650224, Yunnan, China \\ zydyr@163.com
}

\section{Erratum to:}

Chapter 27 in: T. Tan et al. (Eds.)

Advances in Image and Graphics Technologies

DOI: 10.1007/978-981-10-2260-9_27

This paper has been retracted because parts of the work were copied from the following publication: "Automatic Colorization" in http://tinyclouds.org/colorize/. 